\title{
A NOTE ON INDEPENDENCE OF EFFECT ALGEBRAS’ AXIOMATIC SYSTEM
}

\section{IBRAHIM SENTURK and TAHSIN ONER}

Department of Mathematics

Faculty of Sciences

Ege University

Turkey

e-mail: ibrahim.senturk@ege.edu.tr tahsin.oner@ege.edu.tr

\begin{abstract}
In this paper, we mention about the significance of the independence of axiomatic systems and how is obtained. Afterwards, we scrutinize the independence of the axiomatic system of effect algebras which is given by Foulis and Bennett [6].
\end{abstract}

\section{Introduction}

Given an axiomatic system, a natural question arises: are its axioms independent? Such a question is sometimes difficult to answer. For example, the independence of the Axiom of Choice from ZermeloFraenkel set theory's axioms took many decades to be confirmed. Also the very definition of Boolean algebras includes ten axioms and their duals. Reducing the number of axioms requires a great effort, and in this respect Huntington [9, 10] showed that Boolean algebras can be axiomatized by four independent axioms. For another work in this topic,

2010 Mathematics Subject Classification: 06D35, 03C30.

Keywords and phrases: independence, effect algebras, quantum structures.

Received December 19, 2017

(c) 2018 Scientific Advances Publishers 
one can see [15]. We know that a set $S$ of axioms is independent semantically, if none of the elements of $S$ is a consequence of the others. More formally still, the condition is that for every $\varphi \in S$, the sentence $\varphi$ is not a theorem of the theory with axioms $S \backslash\{\varphi\}$. To show independence, it is enough to produce for every $\varphi \in S$ a model $\mathcal{M}_{\varphi}$ in which all the axioms in $S \backslash\{\varphi\}$ are true, but $\varphi$ fails.

We quote Jenčová and Pulmannová's work [11] to state effect algebras that have been introduced by Foulis and Bennett [6]. The effect algebras are a generalization of many structures which arise in the axiomatization of quantum mechanics (Hilbert space effects [14]), orthomodular lattices and posets [1, 16], fuzzy measure theory and manyvalued logic (MV-algebras [3, 4]). Equivalent definitions of effect algebras are provided by Guintini and Greuling [8], and by Kôpka [13]. In this paper, we choose as axioms for effect algebras those given by Foulis and Bennett in [6] and prove that they are independent such as independence of MV-algebras indicated by Cattaneo et al. in [2, 12], respectively.

\section{Preliminaries}

A Boolean algebra can be defined either as a bounded, complemented distributive lattice or as an idempotent ring with unit. Beside this, another equivalent definition can be formulated using only partially defined binary $\oplus$. Then we have the following statement:

Theorem 2.1. Let $(L, \oplus, 0,1)$ be a system consisting of a set $L$ with two special elements $0,1 \in L$ and equipped with a partially defined binary operation $\oplus$ satisfying the following conditions for all $p, q, r \in L:$

(E1) Commutative Law: If $p \oplus q$ is defined, then $q \oplus p$ is defined and $p \oplus q=q \oplus p$.

(E2) Associative Law: If $q \oplus r$ is defined and $p \oplus(q \oplus r)$ is defined, then $p \oplus q$ is defined, and $(p \oplus q) \oplus r$ is defined, and $p \oplus(q \oplus r)=$ $(p \oplus q) \oplus r$. 
(E3) Orthosupplementation Law: For every $p \in L$, there exists a unique $q \in L$ such that $p \oplus q$ is defined and $p \oplus q=1$.

(E4) Zero-One Law: If $1 \oplus p$ is defined, then $p=0$.

(E5) Coherence Law: If $p \oplus q, p \oplus r$, and $q \oplus r$ are defined, then $(p \oplus q) \oplus r$ is defined.

(E6) Law of Compatibility: For all $p, q \in L$; there exist $a, b, c \in L$ such that $b \oplus c$ and $a \oplus(b \oplus c)$ are defined, $p=a \oplus c$ and $q=b \oplus c$.

Then $L$ can be organized into a Boolean algebra in one and only one way so that $0 \leq p \leq 1$ for all $p \in L, p \oplus q$ is defined if and only if $p \wedge q=0$, and if $p \wedge q=0$, then $p \oplus q=p \vee q$. Conversely, if $L$ is a Boolean algebra, and if the partial binary operation $\oplus$ is defined by $p \oplus q=p \vee q$ if and only if $p \wedge q=0$, then $(L, \oplus, 0,1)$ satisfies conditions (E1)-(E6).

Definition 2.1 ([6]). A system $(L, \oplus, 0,1)$ that satisfies conditions (E1)-(E4) in Theorem 2.1 is called an effect algebra.

Definition 2.2 ([6]). Let $L$ be an effect algebra and $p, q \in L$.

(1) We say that $p$ is orthogonal to $q$ and write $p \perp q$ if and only if $p \oplus q$ is defined. If $0 \neq p$ and $p \perp q$, then $p$ is called an isotropic element of $L$.

(2) We say that $p$ is less than or equal to $q$ and write $p \leq q$ if and only if there exists an element $r \in L$ such that $p \perp r$ and $p \oplus r=q$.

(3) The unique element $q$ such that $p \perp q$ and $p \oplus q=1$ is written as $p^{\prime}:=q$ and called the orthosupplement of $p$. 


\section{Independence of the Axioms of Effect Algebra (E1)-(E4)}

In this section, we show that the axiomatic system for effect algebras consists of independent axioms. Therefore, none of these axioms can be removed when constructing any Effect Algebra.

Theorem 3.1. The axioms (E1), (E2), (E3), and (E4) are independent.

Proof. We prove here that the axioms (E1) through (E4) are independent. To see this, we construct a model for each axiom in which that axiom is false while the others are true. Let $\mathcal{M}$ be our model with the universe $U=\{0, x, 1\}$. The symbol $\oplus^{\mathcal{M}}$ is interpreted as the partially defined operation and $0^{\mathcal{M}}, 1^{\mathcal{M}}$ as constants.

\subsection{Independence of (E1)}

The operation $\oplus^{\mathcal{M}}$ is defined in the following table:

\begin{tabular}{c|ccc}
$\oplus$ & 0 & $x$ & 1 \\
\hline 0 & 0 & $x$ & 1 \\
$x$ & 1 & $N E$ & $N E$ \\
1 & 1 & $N E$ & $N E$
\end{tabular}

where NE means: "Not Existing" or "Not Defined". Then we have $a \oplus b \neq b \oplus a$ for all $a, b \in U$ because $x \oplus 0$ is defined and equal to 1 and $0 \oplus x$ is defined but it equals $x$.

For the truth of (E2) let $a, b$, and $c$ be elements in our model $\mathcal{M}$. If $a \oplus(b \oplus c)$ is defined and the result is different from 1 , then $(a \oplus b) \oplus c$ is defined and $a \oplus(b \oplus c)=(a \oplus b) \oplus c$, and the result is $c$. On the other hand, if $a \oplus(b \oplus c)$ is defined and equal to 1 , then $a \oplus(b \oplus c)=(a \oplus b) \oplus c$. Otherwise, we have not existing situation for both sides of identities.

(E2) is true in $\mathcal{M}$. And also it is clear that Orthosupplementation Law and Zero-One Law are true in $\mathcal{M}$. Therefore, (E1) is independent from (E2), (E3), and (E4). 


\subsection{Independence of (E2)}

The operation $\oplus^{\mathcal{M}}$ is defined in the following table:

\begin{tabular}{c|ccc}
$\oplus$ & 0 & $x$ & 1 \\
\hline 0 & $x$ & 0 & 1 \\
$x$ & 0 & 1 & $N E$ \\
1 & 1 & $N E$ & $N E$
\end{tabular}

Since $\left(\mathcal{M}, \oplus^{\mathcal{M}}\right)$ is symmetrical about the main diagonal, $\oplus^{\mathcal{M}}$ is a commutative operation. So, (E1) is true in $\mathcal{M}$. (E3) and (E4) is obtained easily in $\mathcal{M}$. However, $0 \oplus(x \oplus x)$ is defined and equals 1 and also $(0 \oplus x) \oplus x$ is defined but it equals 0 . So, (E2) is not true in $\mathcal{M}$. Therefore, (E2) is independent from the others.

\subsection{Independence of (E3)}

The operation $\oplus^{\mathcal{M}}$ is defined in the following table:

\begin{tabular}{c|ccc}
$\oplus$ & 0 & $x$ & 1 \\
\hline 0 & 0 & $x$ & 1 \\
$x$ & $x$ & $x$ & $N E$ \\
1 & 1 & $N E$ & $N E$
\end{tabular}

(E1) is true in $\mathcal{M}$ because of the main diagonal symmetry. (E2) is obtained in $\mathcal{M}$ by examining all 27 cases. These cases can be resumed as follows:

(i) If the elements $x$ and 1 both occur or 1 occurs more than one in one side of an identity, then we have $N E$ case.

(ii) If the result is defined, then

- it is $x$ if one side of an identity consists only of $x$ 's or only $x$ and 0 occur, otherwise,

- it is 1 if we have only 1 and two 0's, otherwise,

- it is 0 if all the three elements are 0. 
Besides (E4) is also true in $\mathcal{M}$. But for $x \in U$, there is no element $x^{\prime} \in U$ such that $x \oplus x^{\prime}=1$. Therefore, (E3) does not hold in $\mathcal{M}$. Consequently, (E3) is independent from the others.

\subsection{Independence of (E4)}

The operation $\oplus^{\mathcal{M}}$, which is isomorphic to the symmetric group $\mathbb{Z}_{3}$, is defined in the following table:

\begin{tabular}{c|ccc}
$\oplus$ & 0 & $x$ & 1 \\
\hline 0 & 0 & $x$ & 1 \\
$x$ & $x$ & 1 & 0 \\
1 & 1 & 0 & $x$
\end{tabular}

There is a contradiction with (E4) because $x \oplus 1$ exists but $x$ does not equal 0. However, (E1), (E2), and (E3) hold in $\mathcal{M}$.

\section{Conclusion}

As a conclusion, we showed that each axiom is independent from the others in effect algebras axiomatic system. So, any algebra is an effect algebra if and only if it verifies (E1)-(E4) axioms.

\section{References}

[1] E. G. Beltrametti and G. Cassinelli, New Logic of Quantum Mechanics, Cambridge University Press: Cambridge, UK, 2010.

[2] G. Cattaneo and F. Lombardo, Independent axiomatization of MV-algebras, Tatra Mt. Math. Publ. 15(2) (1998), 227-232.

[3] C. C. Chang, Algebraic analysis of many valued logics, Trans. Amer. Math. Soc. 88 (1958), 467-490.

DOI: https://doi.org/10.1090/S0002-9947-1958-0094302-9

[4] R. Cignoli, I. M. L. D’Ottaviano and D. Mundici, Algebraic Foundations of ManyValued Reasoning; Kluwer: Dordrecht, 2000.

[5] A. Dvurečenskij and S. Pulmannová, New trends in quantum structures, Kluwer Acad. Publ.: Ister Sci., Dordrecht, Bratislava, 2000. 
[6] D. J. Foulis and M. K. Bennett, Effect algebras and unsharp quantum logics, Foundations of Physics 24(10) (1994), 1331-1352.

DOI: https://doi.org/10.1007/BF02283036

[7] D. J. Foulis, MV and Heyting effect algebras, Foundations of Physics 30(10) (2000), 1687-1706.

DOI: https://doi.org/10.1023/A:1026454318245

[8] R. Giuntini and H. Greuling, Toward a formal language for unsharp properties, Foundations of Physics 19(7) (1989), 931-945.

DOI: https://doi.org/10.1007/BF01889307

[9] E. V. Huntington, Sets of independent postulates for the algebra of logic, Trans. Amer. Math. Soc. 5(3) (1904), 288-309.

DOI: https://doi.org/10.1090/S0002-9947-1904-1500675-4

[10] E. V. Huntington, New sets of independent postulates for the algebra of logic, Trans. Amer. Math. Soc. 35(1) (1933), 274-304.

DOI: https://doi.org/10.1090/S0002-9947-1933-1501684-X

[11] A. Jencová and S. Pulmannová, Effect algebras with state operator, Fuzzy Sets and Systems 260 (2015), 43-61.

DOI: https://doi.org/10.1016/j.fss.2014.06.002

[12] M. Kolarik, Independence of the axiomatic system for MV-algebras, Mathematica Slovaca 63(1) (2013), 1-4.

DOI: https://doi.org/10.2478/s12175-012-0076-z

[13] F. Kôpka and F. Chovanec, D-posets, Mathematica Slovaca 44 (1994), 21-34.

[14] G. Ludwig, Foundations of Quantum Mechanics, Springer, New-York, 1983.

[15] T. Oner and M. Terziler, Independent of countable sets of formulas of the propositional logic, ARS Combinatoria 12 (2013), 73-80.

[16] P. Pták and S. Pulmannová, Orthomodular Structures as Quantum Logics, Kluwer: Dordrecht, 1991. 